\title{
Analysis of Pseudo-spectral Methods Used for Numerical Simulations of Turbulence
}

\author{
TAPAN K. SENGUPTA ${ }^{1}$, VAJJALA K. SUMAN ${ }^{2,3}$, PRASANNABALAJI SUNDARAM $^{2}$, ADITI \\ SENGUPTA $^{1}$ \\ ${ }^{1}$ Department of Mechanical Engineering, Indian Institute of Technology (ISM) Dhanbad, \\ Dhanbad-826004, INDIA \\ ${ }^{2}$ High Performance Computing Laboratory, Indian Institute of Technology Kanpur, \\ Kanpur-208 016, INDIA \\ ${ }^{3}$ Computational \& Theoretical Fluid Dynamics Division, CSIR-NAL, \\ Bangalore-560017, INDIA
}

\begin{abstract}
Events in turbulent flows computed by direct numerical simulation (DNS) are often calibrated with properties based on homogeneous isotropic turbulence, advanced by Kolmogorov, and given in Turbulence, U. Frisch, Cambridge Univ. Press, UK (1995). However, these computational procedures are not calibrated using numerical analyses in order to assess their strengths and weaknesses for DNS. This is with the exception in "A critical assessment of simulations for transitional and turbulence flows- Sengupta, T.K., In Proc. of IUTAM Symp. on Advances in Computation, Modeling and Control of Transitional and Turbulent Flows, 491-532, (eds. Sengupta, Lele, Sreenivasan, Davidson), WSPC, Singapore (2016)", where such a calibration has been advocated for numerical schemes using global spectral analysis (GSA) for the convection equation. In recent times, due to growing computing power, simulations have been reported using pseudo-spectral methods, with spatial discretization performed in Fourier spectral space and time-integration by multi-stage Runge-Kutta (RK) methods. Here, we perform GSA of Fourier spectral methods for the first time with RK2 and other multistage Runge-Kutta methods using the model linear convection and convection-diffusion equations. With the help of GSA, various sources of numerical errors are quantified. The major limitations of the RK2-Fourier spectral method are demonstrated for DNS and alternate choices are presented. We specify optimal parameters to achieve the best possible accuracy for simulations. There is a one-to-one correspondence of numerical solutions obtained by linear convection-diffusion equation and nonlinear Navier-Stokes equation with respect to numerical parameters. This enables us to investigate the capabilities of the numerical methods for DNS.
\end{abstract}

Key-Words: Computer Simulation, Numerical Simulation, Computer Applications, Pseudo-Spectral Methods, Numerical Methods

Received: March 11, 2021. Revised: October 15, 2021. Accepted: December 16, 2021. Published: January 19, 2022.

\section{Introduction}

Direct numerical simulations (DNS) of turbulent flows have been attempted for decades, with one of the early works being reported [19] for a Reynolds number (based on Taylor's microscale $(\lambda)$ ) of $R_{\lambda}=35$, using a $32^{3}$ computational periodic box. This DNS of three-dimensional (3D) homogeneous isotropic turbulence was compared with the predictions of the direct-interaction turbulence theory. With increase in computing power, the recorded $R_{\lambda}$ increased. Kaneda \& Ishihara [15] reported results showing a $R_{\lambda}=1200$ using a $4096^{3}$ periodic box for $3 \mathrm{D}$ homogeneous isotropic turbulence simulations. These activities progressed to a state where results are reported for $R_{\lambda}=1300$ using a $12288^{3}$ periodic computational box for the simulation of 3D homogeneous isotropic turbulence [5]. A historic account and perspectives of homogeneous isotropic turbulence are detailed in
[13]. The aforementioned simulations for homogeneous isotropic turbulence have been performed using the incompressible Navier-Stokes equation (INSE), given by,

$$
\frac{\partial \mathbf{u}}{\partial t}+\mathbf{u} \cdot \frac{\partial \mathbf{u}}{\partial x}=-\nabla P / \rho+v \nabla^{2} \mathbf{u}+\mathbf{f}
$$

where $\mathbf{u}$ is the solenoidal velocity field, $P$ is the pressure, $\rho$ is the density of the fluid, $v$ is the kinematic viscosity, and $\mathbf{f}$ is a forcing term imposed at a large length scale. Such a forcing term being added to the INSE has been weakly justified as necessary to ensure statistical stationarity of the computed turbulent signal [12] for simulation of homogeneous isotropic turbulence by the RK2Fourier spectral method, as in [5]. This method was originally used in a DNS code [22] with the added forcing term in INSE. The same version of the code continues to be used by a large number of researchers $[14,4,16,6,7,37,11,10,20]$ 
among many others. This approach of using additional forcing has been critiqued [2] for not only this canonical homogeneous problem, but also for so-called DNS of geophysical and engineering flows $[32,3,31]$. For transitional flows, there are many solutions of INSE which do not use such artifices and can be viewed truly as DNS [21, 26, 2].

However, it is noted that such simulations often display issues such as solution "blow-up" after a finite time (especially in the limit of vanishing $v)$. Also, it has been observed that, "experimental measurements in homogeneous turbulence at high Reynolds numbers show the unequivocal presence of a "bottleneck" effect [17]" that has encouraged the authors in the reference to introduce hyperviscosity in the INSE as,

$$
\frac{\partial \mathbf{u}}{\partial t}+\mathbf{u} \cdot \frac{\partial \mathbf{u}}{\partial x}=-\nabla P / \rho+(-1)^{h+1} v_{h} \nabla^{2 h} \mathbf{u}+\mathbf{f},
$$

where $v_{h}$ is the specified constant hyperviscosity coefficient, and $\mathbf{f}$ is the forcing function. The original formulation of the INSE can be retrieved by setting $h=1$ and $\mathbf{f}=0$. It was noted [17] that the "(use of $\mathbf{f}$ is unnatural (as are most other ways of forcing turbulence). However, we are primarily concerned with bottleneck effects on energy spectra, i.e., we investigate one particular characteristic of small-scale turbulence. In this case, use of a large-scale forcing (in order to analyze statistically stationary rather than decaying turbulence) is justifiable on the grounds that the details of the forcing have little effect on the small-scale statistics."

Bracco \& McWilliams [3] simulated the homogeneous stationary 2D turbulence problem by solving the vorticity transport equation given by,

$$
\frac{D \omega}{D t}=D+\mathbf{F},
$$

where $\mathbf{F}$ is, once again, a large scale forcing term, and the other term is given by, $D=\mu \nabla^{-2} \omega+$ $v \nabla^{2} \omega$. The first term in this has been termed by the authors as "physically artificial hypoviscosity". These work on homogeneous, stationary turbulence in 2D and 3D requiring "hypoviscosity" and/ or "hypervisocisity" is an indication that some form of artificiality is being introduced and one is no longer truly solving the INSE. It is to be noted [2] that the time-averaged, compensated spectral density for (i) streamwise, (ii) wall-normal, and (iii) spanwise velocity components plotted as a function of streamwise wavenumber has demonstrated the "bottleneck" effect noted in the experiments [23] for the inhomogeneous flow over a flat plate. In these simulations [2], the INSE has been solved in velocity-vorticity formulation by using high accuracy compact schemes that require fourth diffusion term for numerical stabilization.

There is another aspect of viscous action which is obscured when one inspects periodic flow. It has been demonstrated that the transport equation for enstrophy $\left(\Omega_{1}=\omega_{i} \omega_{i}\right)$ of inhomogeneous flow is given by [30],

$$
\frac{D \Omega_{1}}{D t}=\frac{2}{R e}\left[\frac{1}{2} \nabla^{2} \Omega_{1}-(\nabla \omega)^{2}\right]+2 \omega_{i} \omega_{j} \frac{\partial u_{i}}{\partial x_{j}}
$$

The first set of terms on the right hand side arise due to viscous diffusion. Furthermore, it is to be noted that the first term $\left(\nabla^{2} \Omega_{1}\right)$ will drop out for periodic problems. This has led to a misunderstanding about turbulent flows where diffusion term is negative definite for periodic problems, as in homogeneous isotropic turbulence. The diffusion term is erroneously associated with dissipation, even when the flow is not periodic. The last term on the right hand side of the above equation arises due to vortex stretching, which can act as a production term for enstrophy. However, this term was identified [5] to additionally contribute to self-attenuation in the presence of high nonlinearity during extreme events by creating length scales smaller than the Kolmogorov scale. For Euler equation (in the limit of $R e \rightarrow \infty$ or $v \rightarrow 0$ ) enstrophy was noted to grow unbounded in a finite time [5] citing an observation in Doering [9]. Interestingly, it was furthermore conjectured [5] that such finite time blow-up "would correspond to turbulent solutions of the INSE". At the same time, the authors state that "the question remains open whether the non-linear amplification could overcome viscous damping when the flow is sufficiently turbulent". During transition to turbulence of inhomogeneous 2D flow past different bodies, it has been shown [27] that after flows become fully turbulent following transition, the velocity components always achieve a limiting value, even when vortex stretching is absent.

Having dwelt upon various physical aspects of the flow, one notes that the role of numerical analysis of methods used to compute turbulent flows has received less attention. One such analysis was undertaken by solving the one-dimensional (1D) convection equation, given as [25],

$$
\frac{\partial u}{\partial t}+c \frac{\partial u}{\partial x}=0
$$

In this reference, the Fourier spectral-RK2 method has been shown to be unconditionally unstable. 
Here, we will elaborate on this aspect of oftimplemented numerical methodology for computing turbulence by solving the convection-diffusion equation and associated properties. This equation is more closely tied up with the INSE used to solve turbulent flows, due to the added presence of the diffusion term.

The unknown in GSA is written in hybrid spectral form as $u(x, t)=\int \hat{U}(k, t) e^{i k x} d k$, where $k$ is the wavenumber in $x$-direction. If one starts solving Eq. (4) subjected to the initial condition given by $u\left(x_{j}, t=0\right)=u_{j}^{0}=\int U_{0}(k) e^{i k x_{j}} d k$, then the numerical solution after $n^{\text {th }}$ time-step will be obtained as,

$$
u_{N, j}^{n}=\int U_{0}(k)\left[\left|G_{j}\right|\right]^{n} e^{i\left(k x_{j}-n \phi_{j}\right)} d k
$$

where $G_{j}=\left(\frac{\hat{U}\left(k, t^{n}+\Delta t\right)}{\hat{U}\left(k, t^{n}\right)}\right)$ is the amplification factor and is in general, a complex quantity i.e. $G_{j}=G_{r j}+i G_{i j}$. Its modulus is given by $\left|G_{j}\right|=$ $\left(G_{r j}^{2}+G_{i j}^{2}\right)^{1 / 2}$ and the phase shift per time-step is calculated from, $\tan \phi_{j}=-G_{i j} / G_{r j}$. GSA has been used to develop the error $(e)$ propagation equation for the convection equation given by [29],

$$
\begin{gathered}
\frac{\partial e}{\partial t}+c \frac{\partial e}{\partial x}=-\left[1-\frac{c_{N}}{c}\right] c \frac{\partial \bar{u}_{N}}{\partial x}-\int \frac{V_{g N}-c_{N}}{k}\left[\int i k^{\prime} U_{0}[|G|]^{n} e^{i k^{\prime}\left(x-c_{N} t\right)} d k^{\prime}\right] d k \\
-\int \frac{\operatorname{Ln}|G|}{\Delta t} U_{0}\left[|G|^{n} e^{i k\left(x-c_{N} t\right)} d k\right.
\end{gathered}
$$

Here, the numerical error is given by, $e(x, t)=$ $u(x, t)-\bar{u}_{N}(x, t)$, from which one can derive the governing equation for $e(x, t)$ [29]. The numerical phase speed $\left(c_{N}\right)$ is obtained from $\phi_{j}$ (phase shift per time step), so that $n \phi_{j}=k c_{N} n \Delta t$. The physical phase speed is $c$ for all wavenumbers, but $c_{N}$ is noted to depend on $k$. Thus, the numerical solution is dispersive (in contrast to the non-dispersive nature of $1 \mathrm{D}$ convection equation), and is rewritten as,

$$
\bar{u}_{N}=\int U_{0}(k)[|G|]^{t / \Delta t} e^{i k\left(x-c_{N} t\right)} d k
$$

Having obtained the correct numerical phase speed, the numerical group velocity at the $j^{\text {th }}$ node is expressed as, $\left[\frac{V_{g N}}{c}\right]_{j}=\frac{1}{h N_{c}} \frac{d \phi_{j}}{d k}$. Equation (6) clearly demonstrates that the accuracy of the numerical solution is governed by the terms on the right hand side. From this, we identify $|G|$, $c_{N} / c$ and $V_{g N} / c$ as the main numerical parameters for the convection equation, to determine the error forcing. Such forcing arises due to finite discretizations, which dominates the round-off error as the other contributor. Previously, looking at the linear nature of the governing Eq. (4), researchers assumed that the error dynamics is also dictated by this equation with the right hand side of Eq. (6) going to zero.

It is important to understand the roles played by these numerical parameters in ensuring accuracy of scientific computing. The concept of error propagation was introduced in proper perspective for convection equation [29] and the same has been subsequently shown for the diffusion equation [28] and convection-diffusion equation [33]. GSA has also been used to demonstrate a linear dispersive mechanism for numerical error growth [35]. Recently, GSA has also been used to understand the effect of dispersion and dissipation on both convection and diffusion terms in the 2D linearized compressible Navier-Stokes equation (LCNSE) for hybrid finite difference/Fourier spectral scheme [35].

A major development was achieved [34], where it was shown for the first time that the convectiondiffusion equation truly reflects the dependence on numerical parameters as it does for INSE. This provides the framework to calibrate any numerical method for the solution of INSE (both homogeneous and inhomogeneous flows, with or without periodicity) by checking the numerical parameters to be used in the solution process. The process involves calibration with the convection-diffusion equation which is given by,

$$
\frac{\partial u}{\partial t}+c \frac{\partial u}{\partial x}=v \frac{\partial^{2} u}{\partial x^{2}}
$$

As noted above, in numerical simulations the physical phase speed $(c)$ is altered to the wavenumber-dependent numerical phase speed $\left(c_{N}(k)\right)$ for convection equation. In Eq. (8), the coefficient of diffusion (which is identical to the kinematic viscosity in INSE) also changes from constant $v$ to a wavenumber-dependent numerical coefficient: $v_{N}(k)$. It is customary [24] that, the numerical parameters given by the CourantFriedrich-Lewy (CFL) number $\left(N_{c}=c \Delta t / \Delta x\right)$ and the Peclet number $\left(P e=v \Delta t /(\Delta x)^{2}\right)$ are important in fixing grid spacing $(\Delta x)$ and time step $(\Delta t)$ for solving problems dominated by convection and diffusion. 


\section{Illustrative Simulations of Convection- and Convection-Di.usion Equation}

First, we discretize the governing equation using multi-stage Runge-Kutta time integration [24] and Fourier spectral method for discretizing the convection and diffusion terms as, $\frac{\partial u}{\partial x}=\int i k \hat{U} e^{i k x} d k$ and $\frac{\partial^{2} u}{\partial x^{2}}=\int-k^{2} \hat{U} e^{i k x} d k$. In the computations, these derivatives are evaluated using fast Fourier transform (FFT). From these discrete equations, and using the definition of numerical factors, one obtains the complex parameter $G$ as function of $k \Delta x$ and $N_{c}$ for the convection equation [29]; $k \Delta x$, $N_{c}$ and $P e$ for convection-diffusion equation [33]. Having obtained $|G|$ and $\phi$, one can readily obtain $c_{N} / c$ and $V_{g, N} / c$ for the convection equation. For the convection-diffusion equation, one would also find out $v_{N} / v$, the non-dimensional dispersive coefficient of diffusion [33, 34].

First, we demonstrate solution of convection equation obtained by solving Eq. (6) using Fourier spectral method for spatial discretization and two, three-, and four-stage Runge-Kutta method for time integration. If one chooses a grid spacing of $\Delta x$, then the resolved maximum wavenumber $\left(k_{\max }\right)$ is fixed by the Nyquist criterion, $k_{\max } \Delta x=\pi$. The convection equation is solved in a domain, $0 \leq x \leq 10$ discretized uniformly with 4096 points for the propagation of a wave-packet. The initial condition for the wave packet is given by,

$$
u(x, 0)=e^{-10(x-5)^{2}} \sin \left(k_{0} \Delta x\right)
$$

The wave-packet is characterized by the central wavenumber given by, $k_{0} \Delta x=0.2442$, a value which is significantly lower than the Nyquist limit. This low value ensures that the error sources due to phase error and dispersion error are subdominant as compared to the error created by the $|G|$ term.

In Fig. 1, the propagation of the wave-packet is shown after $30000 \Delta t$, where $\Delta t=4.884 \times 10^{-4}$ is fixed for $N_{c}=0.1$ and $c=0.5$, with RK2 method result shown in the top frame; the three-stage RK (RK3) method result in the middle frame and fourstage RK (RK4) method result depicted in the bottom frame. As the exact solution is simply translated to the right at the speed $c$, one can compare it with the numerical solution. It is evident that RK2-Fourier spectral method results are erroneous. As one is solving a periodic problem, the round-off error magnifies, as determined by $|G|$, and that keeps convecting within the domain accumulating in magnitude. However, no distinguishable errors are noted for the RK3-Fourier spectral and RK4-Fourier spectral methods in Fig. 1 for this low value of CFL number, $N_{c}=0.1$.

In Fig. 2, the propagation of the same wavepacket is shown after a time interval of $30000 \Delta t$, for the same value of $N_{c}=0.1$ with the three time discretization methods used in Fig. 1, with Fourier spectral method for spatial discretization, by solving the convection-diffusion equation. Here, the same time step is used, and the coefficient of diffusion is so chosen that the Peclet number is 0.01 . Unlike the non-dissipative, non-dispersive convection equation case in Fig. 1 for which the physical amplification rate is given by $\left|G_{p h y s}\right|=1$, here for the convection-diffusion equation case the physical amplification would indicate attenuation due to diffusion, such that $\left|G_{P h y s}\right|<1$. In this case, the numerical amplification rate $\left(\left|G_{\text {Num }}\right|\right)$ will be such that $\left|G_{\text {Num }}\right| /\left|G_{P h y s}\right|$ is equal to one for accuracy, and $\left|G_{N u m}\right|>1$ would indicate numerical instability. In Fig. 2, the solutions are shown for $N_{c}=0.1$ and $P e=0.01$ for the three Runge-Kutta methods, with the same wave-packet starting from the same initial locations. All the numerical solutions coincide with the exact solution, implying that the unstable RK2-Fourier spectral method for convection equation has become stable for the convection-diffusion equation for the same value of $N_{c}=0.1$. It has already been shown unambiguously that the numerical behavior of convectiondiffusion equation reflects identically the behavior of the numerical solution of INSE when the numerical parameters are kept identical [34]. For the case of RK3 and RK4 methods, the solutions are error free for these parameter combinations for both the convection- and convection-diffusion equations.

The conditional stability of the RK2-Fourier spectral method for the solution of convectiondiffusion equation, explains its use for the simulation of INSE, despite its failure to do so for the convection equation. These aspects are further explored by solving convection-diffusion equation for a higher CFL number of $N_{c}=0.5$, while keeping the Peclet number at the same level of $P e=0.01$. It is well known [18] that for DNS, the diffusion discretization places a stricter control on the time step via the choice of Peclet number. The solutions obtained by RK2, RK3 and RK4 methods are compared in Fig. 3 for $N_{c}=0.5$ and $P e=0.01$ and the simulations are run only for 101 time-steps. One notes erroneous solutions by the RK2-Fourier spectral method, whereas RK3- and RK4-Fourier spectral methods again reproduce error free propagation of the same wave-packet as before. All of these results shown here indicate the necessity to characterize the space-time dis- 


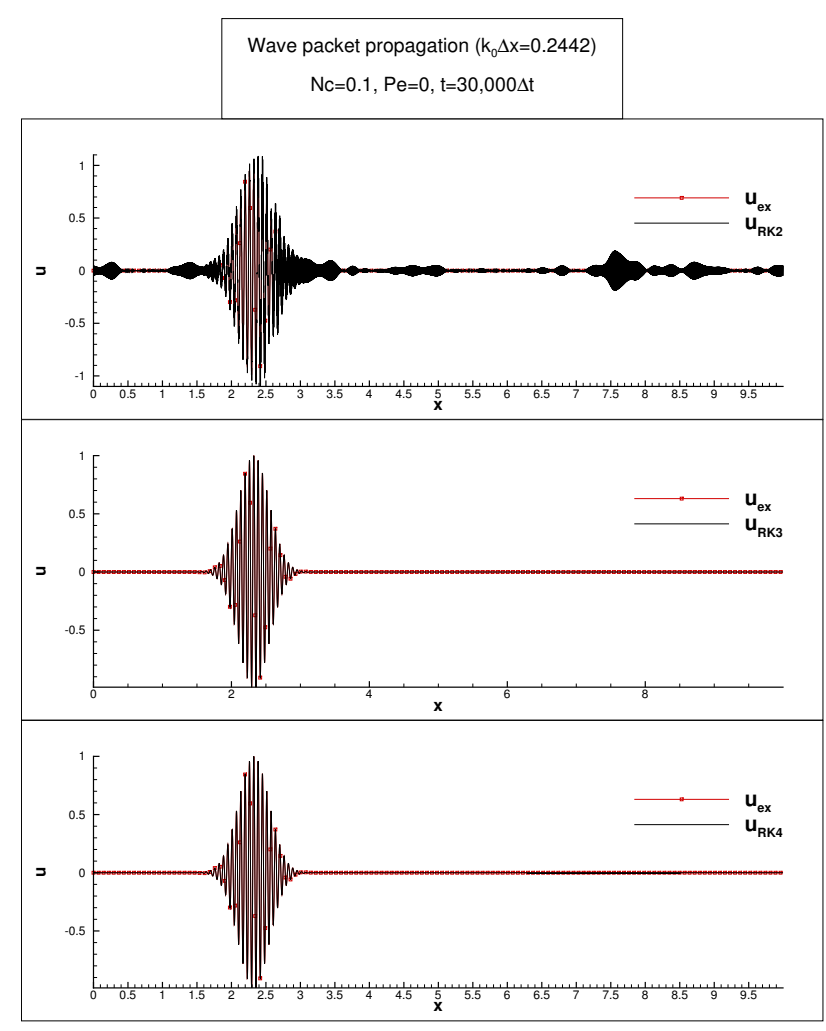

Figure 1: Comparison of numerical solution with exact solution at $t=30,000 \Delta t$ for the convection of a wavepacket using a 1D linear convection equation. Here, Fourier-spectral method is adopted for spatial derivative and RK2, RK3 and RK4 methods are employed for time integration. All simulations are performed on a uniform grid with 4096 points and for a CFL number $N_{c}=0.1$.

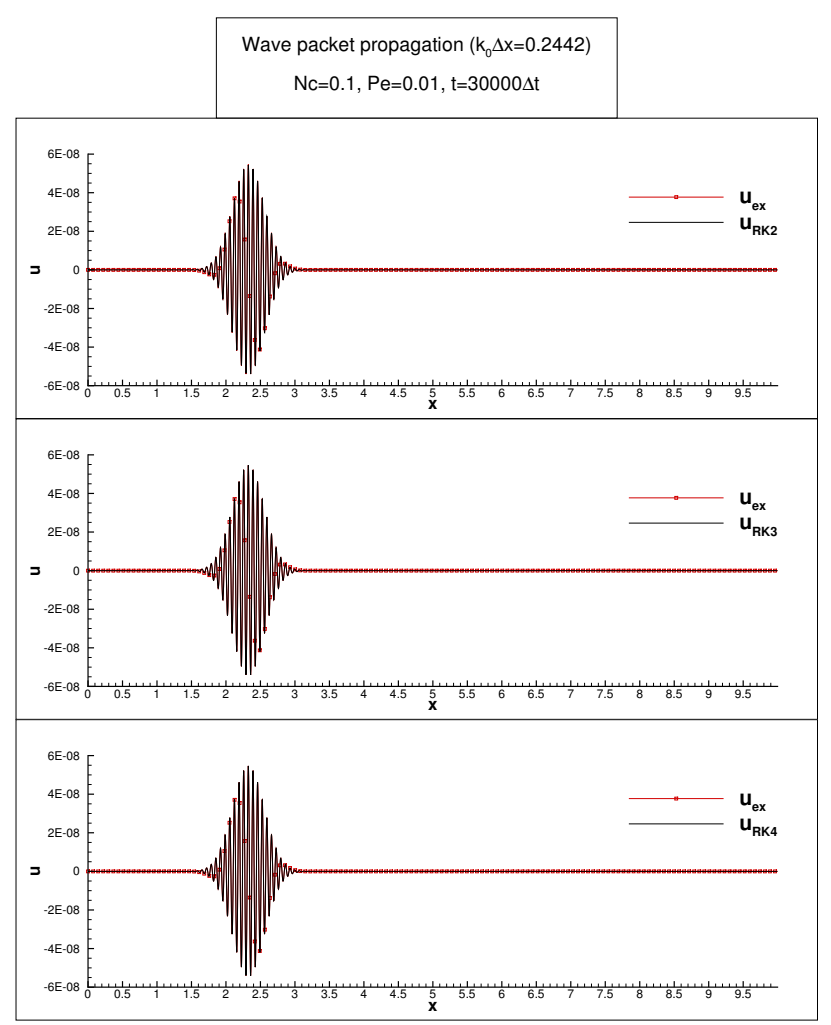

Figure 2: Comparison of numerical solution with exact solution at $t=30,000 \Delta t$ for the convection of a wavepacket using a 1D linear convection-diffusion equation. Here, Fourier spectral method is adopted for spatial derivative and RK2, RK3 and RK4 methods are employed for time integration. All simulations are performed on a uniform grid with 4096 points, with a CFL number $N_{c}=0.1$ and Peclet number $P e=0.01$. 


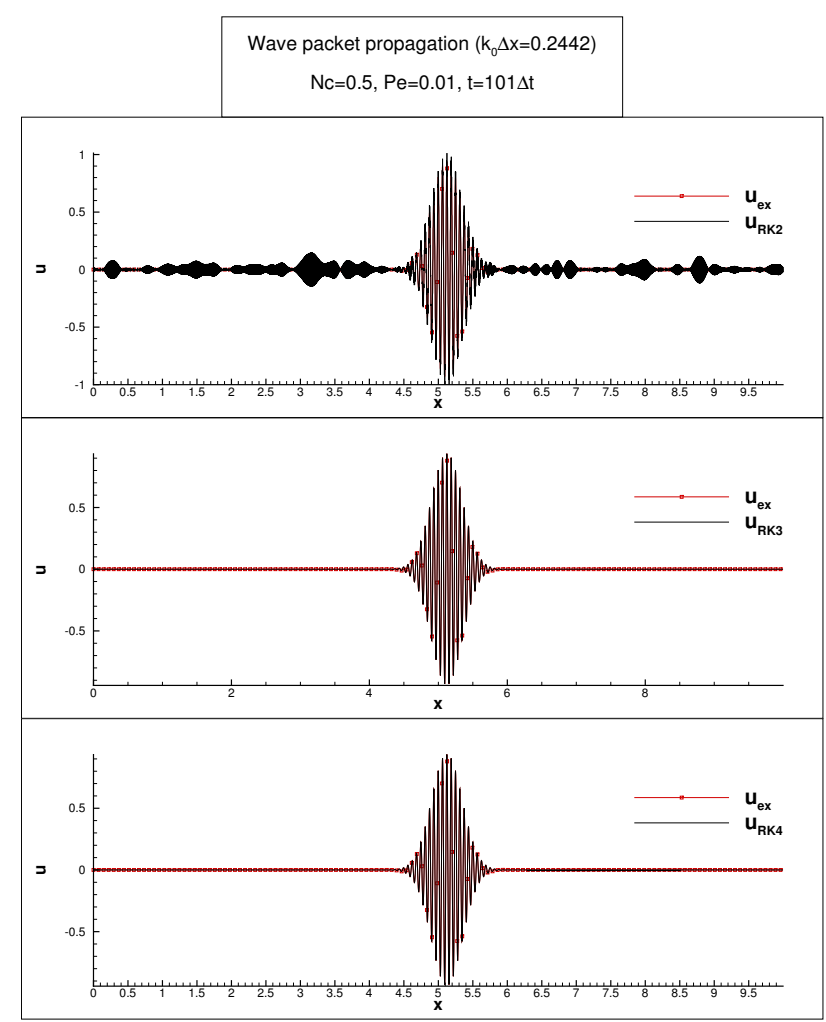

Figure 3: Comparison of numerical solution with exact solution at $t=101 \Delta t$ for the convection of a wavepacket using a 1D linear convection-diffusion equation. Here, Fourier spectral method is adopted for spatial derivative and RK2, RK3 and RK4 methods are employed for time integration. All simulations are performed on a uniform grid with 4096 points, with a CFL number $N_{c}=0.5$ and Peclet number $P e=0.01$. cretization methods. Similar analysis based on the convection equation has been reported in Fig. 1 in [25] for the RK2-Fourier spectral method. It is shown that this method is unconditionally unstable for all CFL numbers for flows dominated by convection. Only for very small values of $k \Delta x$ one observes neutral stability, which can explain why researchers [1] have reported finite-time blow-up for the solution of Euler equation. To understand the implications of the reported results in Figs. 1 to 3 one must perform a thorough study of the numerical properties of convection and convectiondiffusion equations for the pseudo-spectral method proposed in [22] using RK2 time integration. The numerical evidences provided in Figs. 1 to 3 indicate the shortcomings of the RK2-Fourier spectral method. Next, we investigate the possibility of overcoming these by using RK3 and/or RK4 time integration methods.

\section{Error Dynamics for Convection and Convection-Di.usion Equations}

It has been already noted in Eq. (6) that there are three main sources driving the dynamics of error, via the forcing by the phase error term, the dispersion error term and more importantly by numerical instability and unphysical numerical stability term, noted in Eq. (6) for the convection equation. It is a common misconception that numerical stability is desirable [36], which makes many practitioners complacent about this potential source of error, whereas a numerically unstable method is easily recognized due to solution blow-up. For the present analysis of convection and convectiondiffusion equation, numerical parameters are chosen such that the error is solely due to numerical instability and unphysical numerical stability. For the convection equation, an error-free calculation must have neutrally stable numerical amplification factor, $\left|G_{N u m}\right|=1$. In contrast, for convectiondiffusion equation the physical solution attenuates such that $\left|G_{P h y s}\right|<1$ and the numerical solution should reflect this, so that $\left|G_{\text {Num }}\right| /\left|G_{P h y s}\right|=1$. The procedures to calculate these quantities are given for convection [29] and convection-diffusion equations [33], and are provided here in Appendix A.

We use the GSA to map the property charts of the method involving Fourier spectral method for spatial discretization and RK2 method for time integration. We also report the properties at the interior of the domain, as one is interested to analyze the methods to solve 3D homogeneous isotropic turbulence in a periodic box. In Fig. 4, RK2Fourier spectral method is considered for both the canonical equations. For the one-dimensional con- 


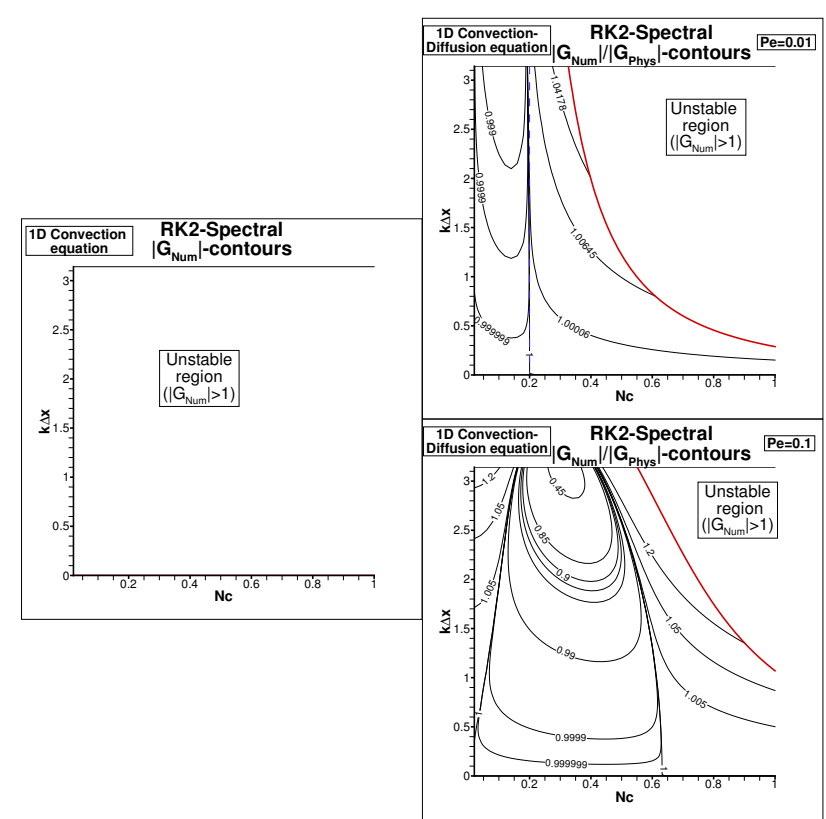

Figure 4: Numerical amplification factor and ratio of numerical amplification factor to the physical amplification factor for the RK2-Fourier spectral scheme plotted in the $\left(N_{c}, k \Delta x\right)$-plane for linear convection and convection-diffusion equations, respectively. For the latter, contours are plotted for two representative Peclet numbers- $P e=0.01$ and 0.1. Dashed line represents the optimal $N_{c}$ value. vection equation, $|G|$-contours are shown plotted in the $\left(N_{c}, k \Delta x\right)$-plane on the left side of Fig. 4. It is noted that barring a small region very close to the origin, the method is unconditionally unstable. This result was also reported [25], and one notes the effects of numerical instability in Fig. 1, in the top frame for which the choice of $N_{c}=0.1$ and $k_{0} \Delta x=0.2442$ does not guarantee stability of all the resolved scales by the Nyquist criterion. The seeds of unstable signals are always present in the round-off error and those wavenumber components interact with the given wave-packet to create multitudes of wave-packets noted for this periodic problem. A non-periodic problem with different inflow and outflow boundary conditions would allow some of the error-packets to leave the computational domain. To emphasize the growth of error that is inherent with the numerical method, a periodic problem is considered so that the signal is always inside the domain, along with the recirculating continuously evanescent error. For the convection-diffusion equation, the error dynamics is additionally affected via a term involving $v_{N} / v$ and the error dynamics is given by,

$$
\begin{aligned}
e_{t}+c e_{x}-v e_{x x}= & \int_{-k_{\max }}^{k_{\max }}\left(v_{N}-v\right) k^{2} e^{-v_{N} k^{2} n \Delta t} U_{0}(k) e^{i k\left(x-c_{N} t^{n}\right)} d k \\
& +\left.i k c_{N} e^{-v_{N} k^{2} n \Delta t} U_{0}(k) e^{i k\left(x-c_{N} t^{n}\right)}\right|_{-k_{\max }} ^{k_{\max }} \\
& -\int_{-k_{\max }}^{k_{\max }}\left(\frac{V_{g N}-c_{N}}{k}\right)\left\{\int_{-k_{\max }}^{k} i k^{\prime} e^{-v_{N} k^{\prime 2} n \Delta t} U_{0}\left(k^{\prime}\right) e^{i k^{\prime}\left(x-c_{N} t^{n}\right)} d k^{\prime}\right\} d k \\
& -\int_{-k_{\max }}^{k_{\max }} i k c e^{-v_{N} k^{2} n \Delta t} U_{0}(k) e^{i k\left(x-c_{N} t^{n}\right)} d k
\end{aligned}
$$

where $v_{N}, c_{N}$ and $V_{g N}$ denote the numerical diffusion coefficient, numerical phase speed and numerical group velocity, respectively. It is interesting to note that the error evolution does not follow the same dynamics as the governing equation. We note that this behaviour was first unequivocally demonstrated for the linear convection equation [29] thus dispelling the earlier held notion that the error evolution always follows the same dynamics as the governing linear equation. Further, it was demonstrated in the same reference that the error due to computing is solely due to three sourcesdiffusion/anti-diffusion, phase and signal propagation, with the errors from each source quanti- fied by the error dynamics equation (given here by Eq. (6)). This is a universal aspect of computing. For the error dynamics equation of convectiondiffusion equation, these three sources of error are also noted. In this paper, we focus solely on the error due to numerical amplification/diffusion for evaluating/analyzing the numerical errors for the Fourier spectral method. Hence, mostly the contours due to $\left|G_{\text {Num }}\right| /\left|G_{\text {Phys }}\right|$ are shown for different simulation cases in the following.

On the right hand side frames of Fig. 4, we have shown the contours of $\left|G_{\text {Num }}\right| /\left|G_{P h y s}\right|$ in $\left(N_{c}, k \Delta x\right)$-plane for $P e=0.01$ and 0.1 . One notices that there is a finite range of $N_{c}$ for which there 


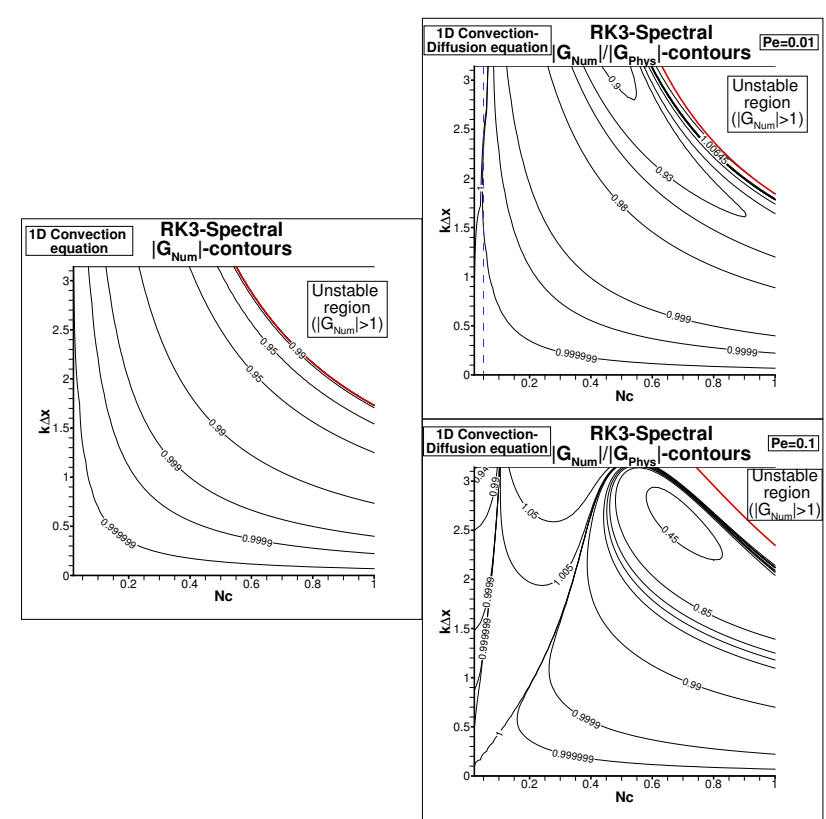

Figure 5: Numerical amplification factor and ratio of numerical amplification factor to the physical amplification factor for the RK3-Fourier spectral scheme plotted in the $\left(N_{c}, k \Delta x\right)$-plane for linear convection and convection-diffusion equations, respectively. For the latter, contours are plotted for two representative Peclet numbers- $P e=0.01$ and 0.1. Dashed line represents the optimal $N_{c}$ value for LES.

is no instability over the full resolved scale. The instability is indicated whenever $\left|G_{N u m}\right|>1$. This is true for both the Peclet numbers, but the contour values in the stable region varies with $k \Delta x$. Presented results here are for $N_{c}=0.1$ and 0.5 , for which the quotient is almost equal to 1 for only a small range of $k \Delta x$ within the Nyquist limit. To find such a value of $N_{c}$ for a chosen $P e$ with maximum range of wavenumbers within the Nyquist limit, one requires to perform GSA.

In Figs. 5 and $6,\left|G_{\text {Num }}\right| /\left|G_{\text {Phys }}\right|$-contours are plotted in $\left(N_{c}, k \Delta x\right)$-plane for $P e=0.0,0.01$ and 0.1 for RK3- and RK4-Fourier spectral methods. Unlike the RK2-Fourier spectral method for the convection equation, RK3 and RK4 time integration methods do not exhibit unconditional numerical instability, as shown in the left panel. However to get neutral stability for this canonical equation, one will be forced to take small value of $N_{c}$, with RK4 method significantly better than the RK3 time integration method allowing much higher values of $N_{c}$. Such values of CFL number will not display finite time blow-up of solution for Euler equation. The right frames in Figs. 5 and $6,\left|G_{\text {Num }}\right| /\left|G_{P h y s}\right|$-contours are plotted in $\left(N_{c}, k \Delta x\right)$ plane for $P e=0.01$ and 0.1 for simulating INSE.

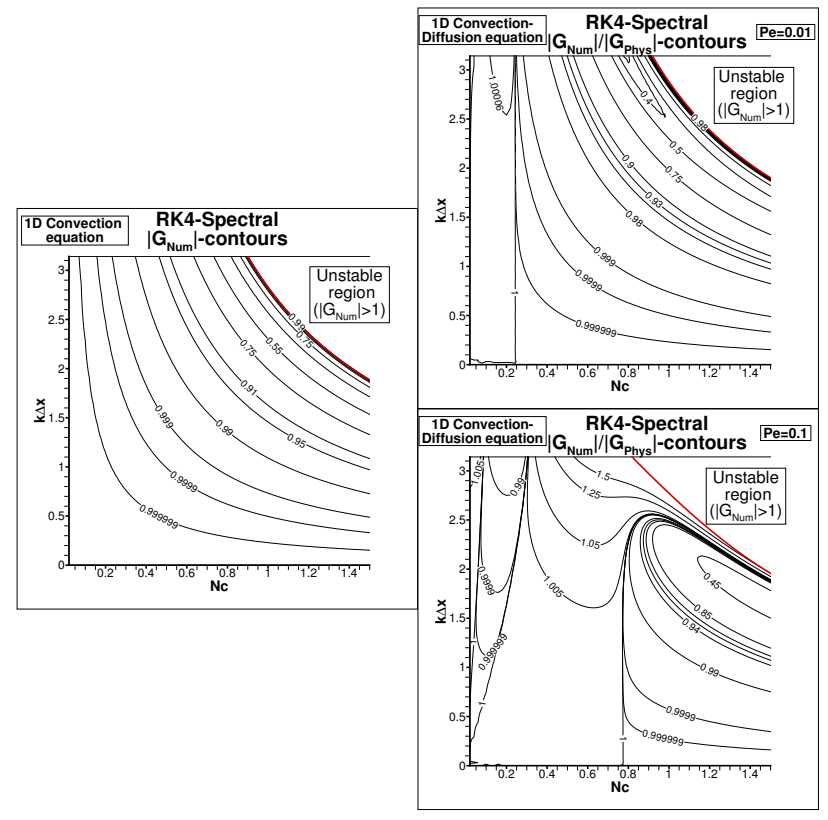

Figure 6: Numerical amplification factor and ratio of numerical amplification factor to the physical amplification factor for the RK4-Fourier spectral scheme plotted in the $\left(N_{c}, k \Delta x\right)$-plane for linear convection and convection-diffusion equations, respectively. For the latter, contours are plotted for two representative Peclet numbers- $P e=0.01$ and 0.1. Dashed line represents the optimal $N_{c}$ value for LES. 


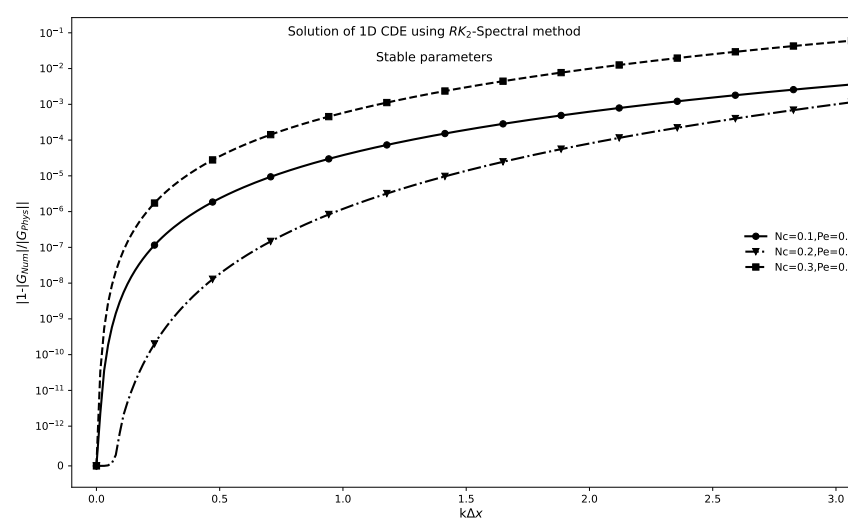

Figure 7: Determination of optimal $N_{c}$ for accurate solution of $1 \mathrm{D}$ convection-diffusion equation using RK2-Fourier spectral method for $P e=0.01$. Accuracy is evaluated with respect to error in numerical amplification factor $\left(\left|1-\left(\left|G_{\text {num }}\right| /\left|G_{\text {phys }}\right|\right)\right|\right)$ for all resolvable wavenumbers $(k \Delta x)$.

For RK3 time integration method, performing accurate simulation would require taking vanishingly small values of $N_{c}$, for vanishingly small values of $P e$. It must however, be remembered that for no parameter combinations, one will reproduce the exact solution for the fully resolved Nyquist limit of wavenumbers $(k \Delta x=\pi)$. For the RK4 time integration method, the permissible values of $N_{c}$, $P e$ for performing near-DNS simulation will be relatively higher than that will be obtained by the RK3 time integration method. Such imperfectly resolved computations can be viewed more as an implicit large eddy simulation. Overall, comparing the $\left|G_{\text {Num }}\right| /\left|G_{P h y s}\right|$-contours in Figs. 4 to 6 , it appears that RK4-Fourier spectral method will provide the best results for $P e=0.01$ and $N_{c}=0.243793$, as described next.

In Figs. 4 to 6 , one can note the contribution to error dynamics by the term involving $\left|G_{N u m}\right| /\left|G_{P h y s}\right|$ for specific values of $P e$. On a cursory observation, one notes that for a specific value of $N_{c},\left|G_{N u m}\right| /\left|G_{P h y s}\right|$ value keeps changing with $k \Delta x$. To quantify such variations and to obtain an optimal value of $N_{c}$ for which the error will be least due to this $\left|G_{\text {Num }}\right| /\left|G_{\text {Phys }}\right|$-term in Fig. 7, we have plotted ||$G_{\text {Num }}|/| G_{P h y s}|-1|$ as a function of $k \Delta x$ for the RK2-spectral method showing that the optimal error forcing is obtained for $N_{c}=0.2$, which is shown to be lower than that for $N_{c}=0.1$ and 0.3 . Having noted this optimal value of $N_{c}=0.2$, one also notices that the forcing by this term increases monotonically in nonlinear manner with increase in wavenumber up to the Nyquist limit. Such monotonic growth in error with wavenumber is clearly apparent from Fig. 4, for these lower

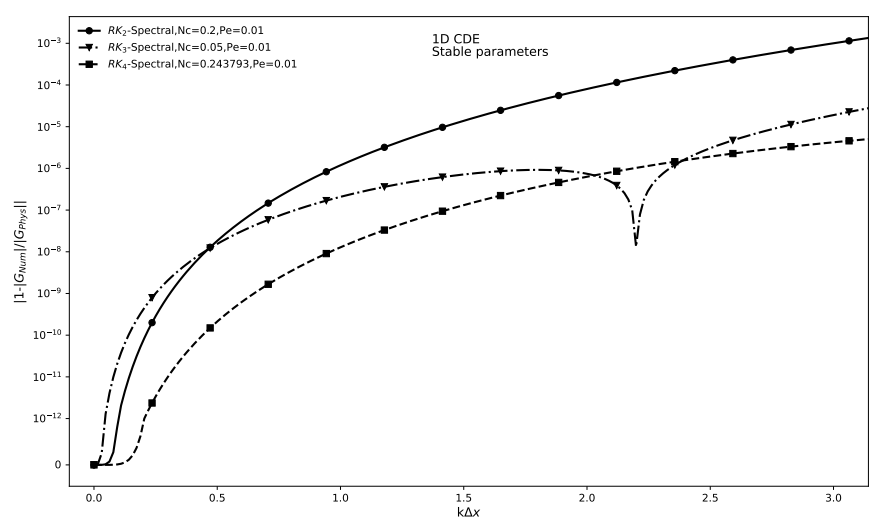

Figure 8: Comparison of the accuracy of RK2, RK3 and RK4 time integration schemes in solving the 1D convection-diffusion equation for $P e=0.01$ using Fourier spectral method. Accuracy is evaluated with respect to error in numerical amplification factor $\left(\left|1-\left(\left|G_{\text {num }}\right| /\left|G_{\text {phys }}\right|\right)\right|\right)$ for all resolvable wavenumbers $(k \Delta x)$. Presented values of $N_{c}$ correspond to the respective optimal values of the time integration schemes.

values of CFL, which is also restricted to the right due to numerical instability for the choice of $N_{c}$. Even though the numerical instability region reduces with increasing $P e$, the higher error with increase in wavenumber is drastic.

Having noted the existence of optimal $N_{c}$ value for RK2-Fourier spectral method as equal to 0.2 , next we locate the same for RK3-Fourier spectral and RK4-Fourier spectral methods. The variation of contributions by the optimal $N_{c}$ choices to error dynamics is compared in Fig. 8. In Fig. 5, the variation of $\left|G_{\text {Num }}\right| /\left|G_{\text {Phys }}\right|$-contours in $\left(N_{c}, k \Delta x\right)$ plane indicates that the error dynamics term dependent on $\left|G_{\text {Num }}\right| /\left|G_{\text {Phys }}\right|$ is more complex and contribute higher values. For the RK3-Fourier spectral method such an optimal value is noted for $N_{c}=0.05$ and for RK4-Fourier spectral method the optimal error is obtained for $N_{c}=0.243793$ for the choice of $P e=0.01$ with the results depicted in Fig. 8. The quantitative variation of error forcing for RK3-spectral method in this figure was shown qualitatively in Fig. 5. But, the RK4-Fourier spectral method shows monotonic variation of $\left|G_{\text {Num }}\right| /\left|G_{P h y s}\right|$ with wavenumber, that causes the least error among these three time integration methods.

\section{Evaluating Schemes for High Reynolds Number Computations}

In this section, we analyze the performance of RK2, RK3 and RK4 time integration schemes with the Fourier spectral spatial discretization for high 
Reynolds number flow computations. Such computations imply very small values of $P e$, as $v$ is equal to $\frac{1}{R e}$. Hence, GSA for low values of $P e$ is warranted as it will reveal the performance in terms of accuracy of the schemes, and in turn can be used to pinpoint the causality between the error and the chosen numerical parameters. Furthermore, this analysis will provide information to all to make informed choices for the parameters to attain a level of desired accuracy.

In Figs. 9 and 10, contours of $\left|G_{\text {Num }}\right| /\left|G_{\text {Phys }}\right|$ are plotted for the RK2- and RK4-Fourier spectral methods, respectively. In these figures, the left frame corresponds to the properties for the 1D convection equation, whereas the right frames are for the convection-diffusion equation with low values of $P e=0.001$ and 0.0001 . As noted earlier, the RK2-Fourier spectral method is unconditionally unstable for the $1 \mathrm{D}$ convection equation, whereas it is conditionally stable for the convection-diffusion equation. From Fig. 9, one notes the stable region of the RK2-Fourier spectral scheme to decrease with decreasing $P e$. For $P e=0.001$, numerical instability is noted for $N_{c} \geq$ 0.175 , which is the critical limit i.e. $N_{c_{c r}}=0.175$. This reduces to $N_{c_{c r}}=0.096$ for a reduced $P e=$ 0.0001 . For a higher $P e=0.01$, one notes $N_{c_{c r}}=$ 0.33 . Thus, $N_{c_{c r}}$ decreases with decrease in $P e$. For the RK4-Fourier spectral method, $N_{c_{c r}}=0.905$, $0.905,0.92$ for $P e=0.0001,0.001$ and 0.01 , respectively. This, once again, shows that the region of stability decreases with decrease in $P e$. This is a real characteristic of any numerical scheme for which adding diffusion (either numerical or physical) stabilizes it, as long as $P e$ value remains below a critical value. It is noted that while the $N_{c_{c r}}$ reduces to very low values for the RK2 scheme, a marginal reduction is noted for the RK4 scheme. This can be explained by the lowest bound on $N_{c_{c r}}=0$ being set for the convection equation $(P e=0)$, for which the RK2 scheme is unconditionally unstable. But the RK4 scheme does not suffer from unconditional instability and the reduction in $N_{c_{c r}}$ is minimal. For $P e \neq 0$, a stabilizing effect overcomes the unconditional instability of the RK2 scheme with $N_{c_{c r}}$ increasing. Thus, RK2Fourier spectral method is unsuitable for computing high Reynolds number flows.

As noted earlier from the analysis of error evolution and its propagation given by Eqn. (10) for the convection-diffusion equation, all numerical schemes must satisfy zero diffusion error i.e. $\left|G_{\text {Num }}\right| /\left|G_{P h y s}\right|$ is equal to 1 for the resolved wavenumbers. Thus, this is a necessary condition to be satisfied by every scheme seeking to obtain accurate results and therefore, qualify for

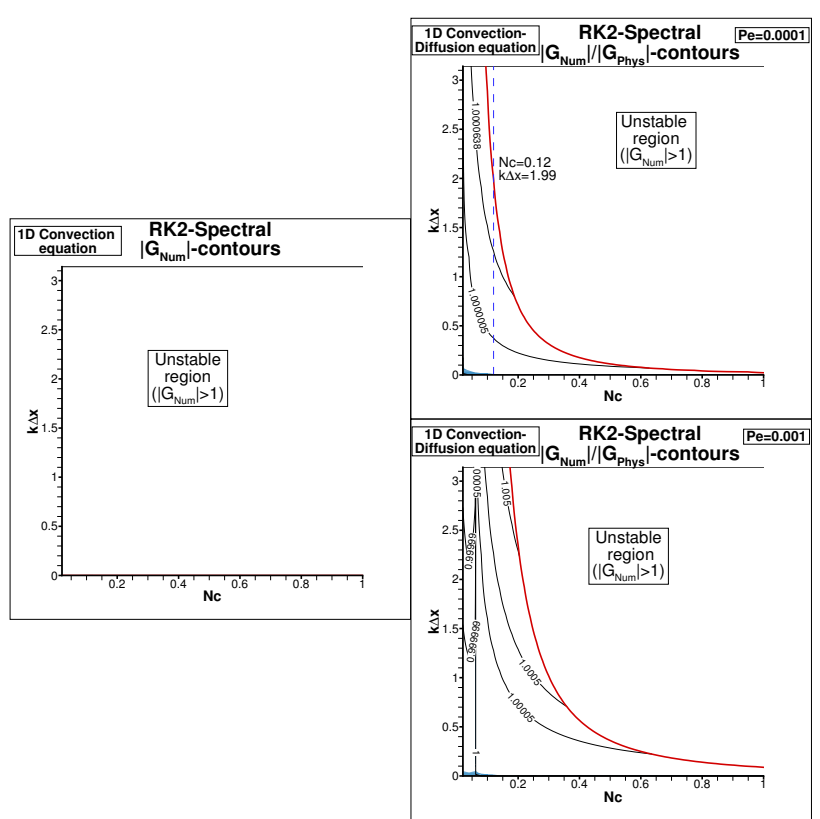

Figure 9: Numerical amplification factor and ratio of numerical amplification factor to the physical amplification factor for the RK2-Fourier spectral scheme plotted in the $\left(N_{c}, k \Delta x\right)$-plane for linear convection and convection-diffusion equations, respectively. For the latter, contours are plotted for two representative Peclet numbers- $P e=0.0001$ and 0.001 . Dashed line represents the conditions at which numerical simulations are performed. Blue coloured region indicates a zone with zero diffusion error.

performing strict DNS. In Figs. 9 and 10, the region with zero diffusion error is shown marked by blue colour. It is interesting to note that while RK4 scheme is superior to RK2 due to the larger extent of zero diffusion error, strict DNS is impossible as one does not obtain zero error at all resolved wavenumbers.

Next, the property charts obtained earlier are corroborated by computing the propagation of the wave-packet using RK2-, RK3- and RK4schemes with the Fourier pseudo-spectral method. The computations are performed with uniformly spaced grids having 4096 points in a domain $x \sim$ $[0,10]$, considered in section 2 . The packet is centered around $k \Delta x=0.22$ and time step is chosen as $5.86 \times 10^{-4}$ such that $N_{c}=0.12$ with $c=0.5$. The diffusion coefficient is determined from $P e=$ 0.0001 . Figure 11 shows the solutions plotted after 30,000 $\Delta t$ for RK2-, RK3- and RK4-Fourier pseudo-spectral methods, respectively. While this parameter combination is noted to be stable for the RK3-and the RK4-methods, the RK2method suffers a numerical instability due to antidiffusion, as indicated by a dashed vertical line 


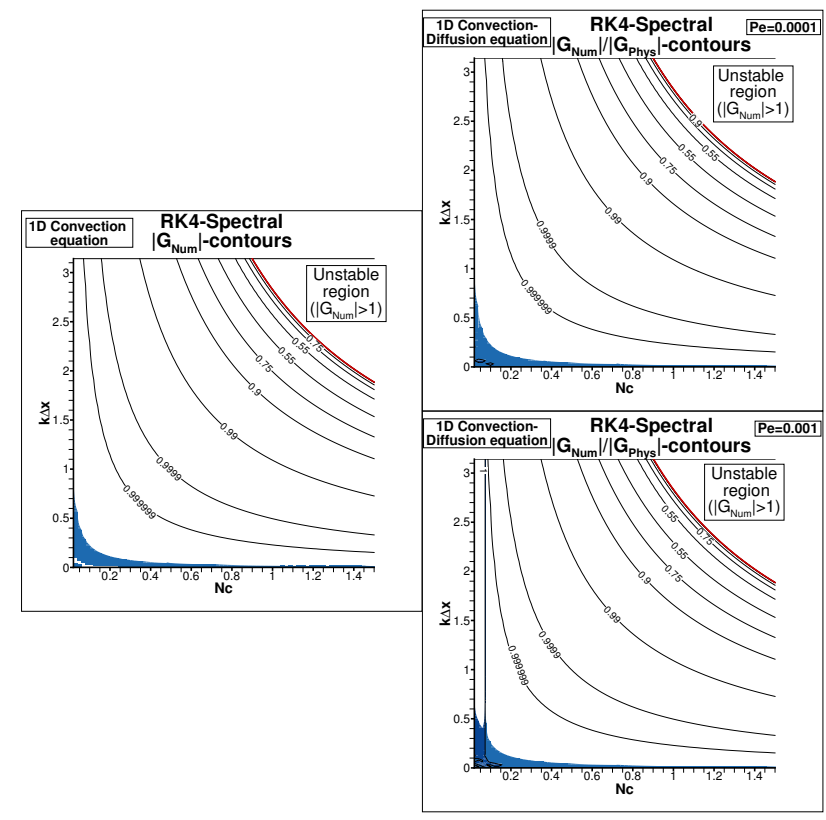

Figure 10: Numerical amplification factor and ratio of numerical amplification factor to the physical amplification factor for the RK4-Fourier spectral scheme plotted in the $\left(N_{c}, k \Delta x\right)$-plane for linear convection and convection-diffusion equations, respectively. For the latter, contours are plotted for two representative Peclet numbers- $P e=0.0001$ and 0.001 . Blue coloured region indicates a zone with zero diffusion error.

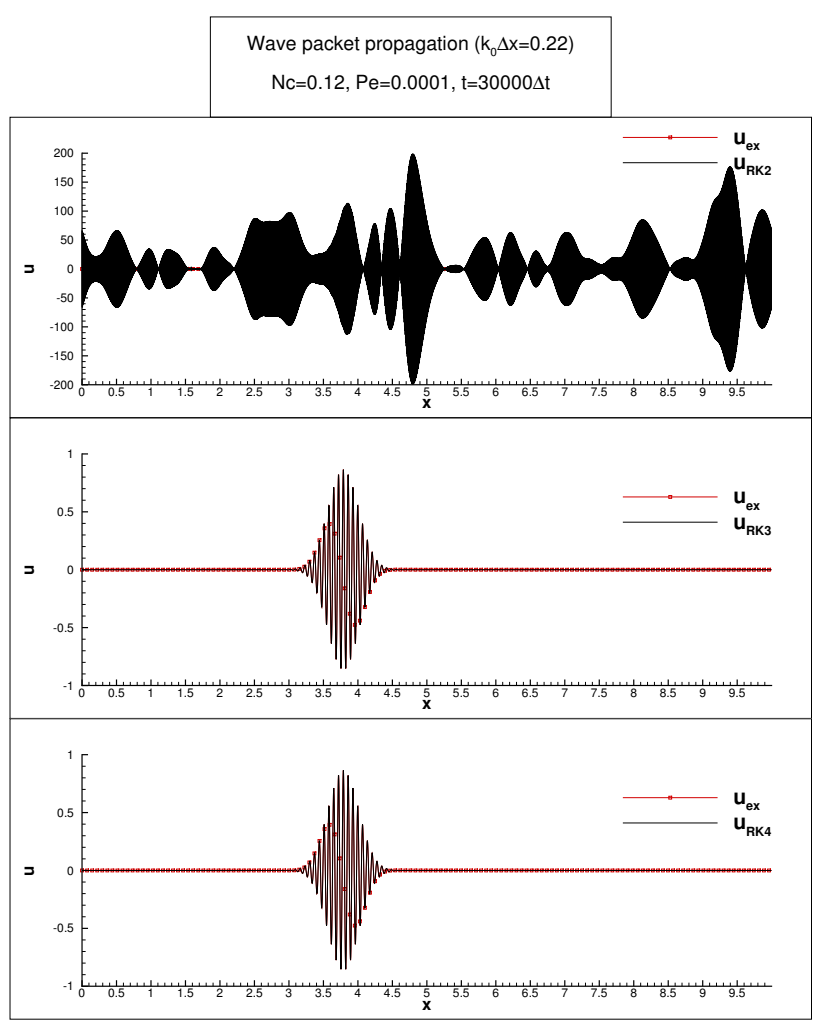

Figure 11: Comparison of numerical solution with exact solution for a time $t=30,000 \Delta t$ for the convection of a wavepacket using a 1D linear convectiondiffusion equation. Here, Fourier spectral method is adopted for spatial derivative and RK2, RK3 and RK4 methods are employed for time integration. All simulations are performed on a uniform grid with 4096 points, with a CFL number $N_{c}=0.12$ and Peclet number $P e=0.0001$.

(blue) in Fig. 9. From this we note that instability occurs for $k \Delta x \geq 1.99$. The computation validates the observations from the analysis. Results for the RK3- and RK4-methods show that the numerical solution follows the exact solution accurately, whereas the solution obtained by RK2-method is noted with unphysical wave-packets and high amplitudes attributed to anti-diffusion.

One notes that although the wave-packet is centered at $k \Delta x=0.22$, the computation with RK2 time integration shows instabilities. The explanation for this behavior is noted from the analytical structure of the wavepacket which results in a spectrum in the Fourier space containing contributions from all wavenumbers upto the Nyquist limit. These high wavenumber amplitudes, despite having nearly negligible amplitudes initially, are amplified by $\left|G_{\text {num }}\right|$ every time-step to cause exponential growth leading to numerical instability and eventual blow-up (not shown). We also 


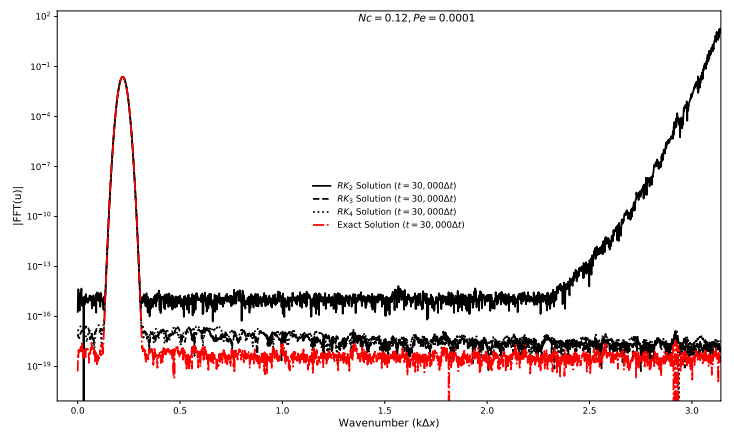

Figure 12: Comparison of FFT of the exact solution with solution obtained from RK2, RK3 and RK4 time integration schemes in solving the 1D linear convection-diffusion equation for Peclet number 0.0001 and $N_{c}=0.12$ using Fourier spectral method.

clear a misconception about the necessity of the presence of relevant wavenumbers for the instability to occur. While it is intuitive to assume that instabilities will only occur if the relevant wavenumbers are present, practical computations suffer from random/round-off errors which excite all scales and thus, the seeds for instability are created. Therefore, it is not necessary for the relevant wavenumbers to be present in the initial solution for the instability to occur. Round-off errors inherent in any computing will trigger the unstable scales, leading to eventual instabilities.

In Fig. 12, the FFT of the numerical solution at $t=30,000 \Delta t$ is plotted for the numerical solutions obtained with RK2-, RK3- and RK4-Fourier spectral methods. In the same figure, the spectrum of the exact solution is also shown for reference. The scale selection for instability for RK2-Fourier spectral method is evident by noting the increasing amplitudes of the high wavenumber components which corroborates very well with the vertical dashed blue line in Fig. 9. It is interesting to note for the RK2-Fourier spectral method that the amplitudes around the central wavenumber of the wave packet are accurately represented with respect to the reference analytical solution whereas amplitudes for $k \Delta x \geq 2.2$ show a visible departure. The solutions due to RK3- and RK4-Fourier spectral methods show a very good comparison with the reference solution. This behaviour is consistent with GSA.

These results clearly demonstrate the utility of GSA which enables an accurate characterization of the behavior of numerical methods in solving problems involving convection and convection-diffusion processes. Furthermore, these analyses have a di- rect bearing on the accuracy of Navier-Stokes simulations due to their one-to-one correspondence with linear convection-diffusion equation [34].

\section{Summary and Conclusions}

A detailed analysis and calibration is performed using global spectral analysis (GSA) for the numerical methods employing Fourier spectral and multistage Runge-Kutta (RK) methods. In particular the RK2 time integration method is studied to assess the schemes for high accuracy simulations, as it has been proposed as DNS over the last five decades $[19,12,22,5]$. These pseudo-spectral schemes are analyzed here for the linear convection and linear convection-diffusion equations as these serve as appropriate model equations for assessing and calibrating numerical errors for Euler and Navier-Stokes equation governed flows, respectively. In particular, the analysis based on the latter is important due its one-to-one correspondence with the incompressible Navier-Stokes equation [34]. With the help of GSA, the schemes' diffusion, dispersion and signal propagation properties are accurately predicted. To our knowledge such results have not been reported before.

Computations and numerical analysis presented here confirm the unconditional numerical instability of RK2-Fourier spectral method in solving the $1 \mathrm{D}$ convection equation, as reported also by [25]. Hence, this method is unsuitable for simulating inviscid problems such as the Euler equation which is often noted in the literature [1]. The Fourier spectral- RK2 method displays stability for the solution of convection-diffusion equation for Peclet value 0.01, shown in Fig. 2 and analysed with results in Fig. 4. In comparison, RK3 or RK4 time integration schemes used with the Fourier spectral method shows numerical stability for both the convection and convection-diffusion equations, demonstrated by Figs. 1-3, 5 and 6 . Thus, choosing a higher order integration scheme instead of RK2, leads to the elimination of numerical instability.

Error dynamics analysis performed in section 3 help answer the question of which among the RK2, RK3 and RK4 methods offer best accuracy for the Fourier spectral method in solving the convectiondiffusion equation. Although Eqn. (10) identifies all sources of numerical error, here only the error contributed by the schemes' inability to represent diffusion accurately is used as the necessary condition for high accuracy computations. This contribution is assessed by noting the difference between numerical and physical diffusion, which is also the difference between numerical amplification and physical amplification factors, repre- 
sented by $|1-| G_{\text {Num }}|/| G_{P h y s}||$. From such analysis, optimal $N_{c}$ values are determined for minimum error in diffusion. Comparing RK2, RK3 and RK4 time integration methods for a fixed Peclet number for optimal $N_{c}$, the RK4 method shows superior accuracy in Fig. 8. For RK2-Fourier spectral method the optimal $N_{c}=0.2$ is for $P e=0.01$, whereas for the RK3- and RK4-Fourier spectral methods optimal $N_{c}$ values are 0.05 and 0.243793 , respectively. This conclusively demonstrates that RK4 time integration offers the best accuracy for Fourier spectral method among these time integration schemes.

More importantly, the analysis reveals that strict DNS is impossible as all the schemes do not display vanishing $|1-| G_{\text {Num }}|/| G_{P h y s}||$ for the resolved wavenumbers for any choice of $N_{c}$. Thus, the optimal parameter noted above are for underresolved DNS only. The best Fourier spectralRK4 method in Fig. 10, shows exact vanishing of $|1-| G_{N u m}|/| G_{P h y s}||$ for a range of $N_{c}$ as given by the blue coloured region with the maximum limit on accurately resolved wavenumbers $(k \Delta x=0.8$ for $\mathrm{Pe}=0.0001$ and $k \Delta x=0.6$ for $\mathrm{Pe}=0.001)$ noted at lower $N_{c}$ values.

GSA performed for the RK2-Fourier spectral method for Peclet number $P e=0.01$ in Fig. 13 shows the diffusion, dispersion and signal propagation errors for the method. The latter two quantities are the sufficient conditions used for evaluting the accuracy of the method. For the optimum $N_{c}$ value determined using the necessary condition for the RK2 method, a maximum of $5 \%$ and $15 \%$ errors are noted for the phase speed and signal propagation, respectively. Thus, one notes that the sufficient condition too is not satisfied at all resolvable wavenumbers.

The reduced stability of RK2-Fourier spectral method used for solving Navier-Stokes equations at high Reynolds numbers by reducing the time step (i.e. by decreasing Peclet number) is a counter-intuitive result reported here for the first time. As Peclet number is decreased, the stability region decreases rapidly as demonstrated in Fig. 9, rendering the method unsuitable unless other non-physical fixes are employed to suppress numerical instability. The error due to dispersion shown in Fig. 13 negates the use of RK2-Fourier spectral method completely for DNS. The analyses shows that higher order RK schemes can alleviate the instability problem of the RK2-Fourier spectral method, but even then the higher order time integration schemes are strictly incapable of performing DNS.

Further evidence of the agreement between GSA and numerical simulations is shown in Figs.

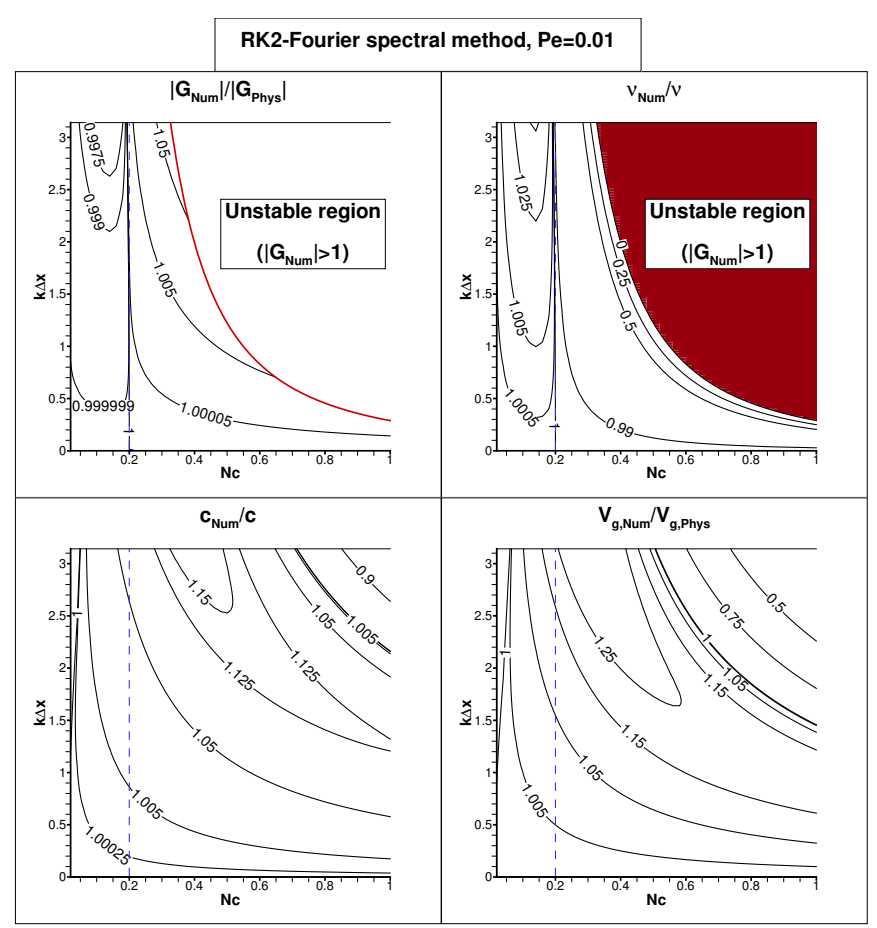

Figure 13: GSA analysis of RK2-Fourier spectral method for the 1D convection-diffusion equation plotted in the $\left(N_{c}, k \Delta x\right)$-plane for $P e=0.01$. Plotted are the quantities of ratios of numerical amplification to physical amplification factor $\left(\left|G_{N u m}\right| /\left|G_{P h y s}\right|\right)$, numerical diffusion to physical diffusion coefficient $\left(v_{N u m} / v\right)$, numerical phase speed to physical phase speed $\left(c_{N u m} / c\right)$ and numerical group velocity to physical group velocity $\left(V_{g, \text { Num }} / V_{g, \text { Phys }}\right)$. 
11 and 12, to detect instability and its scale selection. This is demonstrated with an initial solution containing unstable wavenumbers for early time explosion of numerical solution. It is not necessary to use such initial condition for instability, as the round-off error inherent in computing, will have the seed of the unstable wavenumber, capable of triggering the eventual instability in long time solution.

The present work shows unequivocally that RK3, RK4 time integration schemes are mandatory in using Fourier spectral method for spatial discretization in order to ensure the necessary condition of numerical stability for high Reynolds number flow computations. For required accuracy in DNS, one must thereafter investigate for the sufficient condition of ensuring negligible dispersion error, as given in Eqn. (10). In future, detailed analysis considering errors due to phase and signal propagation will be performed in order to fine tune the simulation parameters for both the necessary and sufficient conditions.

\section{Declaration of Interests}

The authors report no conflict of interest.

\section{References:}

[1] Beale J. T., Kato T., \& Majda A., Remarks on the breakdown of smooth solutions for the 3-D Euler equations, Commun. Math. Phys., 94, 61-66 (1984)

[2] Bhaumik S., \& Sengupta T. K., Precursor of transition to turbulence: Spatiotemporal wave front, Phys. Rev. E, 89(4), 043018 (2014)

[3] Bracco A., \& McWilliams J. C., Reynoldsnumber dependency in homogeneous, stationary two-dimensional turbulence, J. Fluid Mech., 646, 517-526 (2010)

[4] Buaria D., Bodenschatz E., \& Pumir A., Vortex stretching and enstrophy production in high Reynolds number turbulence, Phys. Rev. Fluids, 5(10), 104602 (2020)

[5] Buaria D., Pumir A. \& Bodenschatz E., Selfattenuation of extreme events in Navier-Stokes turbulence, Nat. Commun., 11, 5852 (2020)

[6] Buaria D., \& Sreenivasan K. R., Dissipation range of the energy spectrum in high Reynolds number turbulence, Phys. Rev. Fluids, 5(9), 092601 (2020)

[7] Choi Y., Kim B., \& Lee C., Alignment of velocity and vorticity and the intermittent distribution of helicity in isotropic turbulence, Phys. Rev. E. 80(1), 017301 (2009)
[8] David Cl., Sagaut P., \& Sengupta T., A linear dispersive mechanism for numerical error growth: spurious caustics, Euro. J. Mech. B/Fluids, 28(1), 146-151 (2009)

[9] Doering C. R., The 3D Navier-Stokes problem, Ann. Rev. Fluid Mech., 41, 109-128 (2009)

[10] Donzis D. A., \& Yeung P. K., Resolution effects and scaling in numerical simulations of passive scalar mixing in turbulence, Physica D: Nonlinear Phenomena, 239(14), 1278-1287 (2010)

[11] Donzis D. A., Yeung P. K., \& Sreenivasan K. R., Dissipation and enstrophy in isotropic turbulence: Resolution effects and scaling in direct numerical simulations, Phys. Fluids. 20(4), 045108 (2008)

[12] Eswaran V., \& Pope S. B., An examination of forcing in direct numerical simulations of turbulence, Computers \& Fluids, 16(3), 257-278 (1988)

[13] Frisch U., Turbulence: The Legacy of A. N. Kolmogorov, Cambridge Univ. Press, UK (1995)

[14] Ishihara T., Gotoh T., \& Kaneda Y., Study of high-Reynolds number isotropic turbulence by direct numerical simulation, Ann. Rev. Fluid Mech., 41, 165-180 (2009)

[15] Ishihara T., Morishita K., Yokokawa M., Uno A. \& Kaneda Y., Energy spectrum in high-resolution direct numerical simulations of turbulence, Phys. Rev. Fluids, 1(8), 082403 (2016)

[16] Kaneda Y., Ishihara T., Yokokawa M., Itakura K., \& Uno A., Energy dissipation rate and energy spectrum in high resolution direct numerical simulations of turbulence in a periodic box, Phys. Fluids, 15(2), L21-L24 (2003)

[17] Lamorgese A. G., Caughey D. A., \& Pope S. B., Direct numerical simulation of homogeneous turbulence with hyperviscosity, Phys. Fluids, 17(1), 015106 (2005)

[18] Moin P., \& Mahesh K., Direct numerical simulation: A tool in turbulence research, Ann. Rev. Fluid Mech., 30, 539-578 (1998)

[19] Orszag S., \& Patterson G., Numerical Simulation of three-dimensional homogeneous isotropic turbulence, Phys. Rev. Lett., 28(2), 76-79 (1972) 
[20] Ranjan A., \& Davidson P. A., DNS of a buoyant turbulent cloud under rapid rotation, in IUTAM Symp. Proc. Advances in Computation, Modeling and Control of Transitional and Turbulent Flows, 452-460 (2016)

[21] Rist U., \& Fasel H., Direct numerical simulation of controlled transition in a flat-plate boundary layer, J. Fluid Mech., 298, 211-248 (1995)

[22] Rogallo R. S., Numerical experiments in homogeneous turbulence, NASA Tech. Memo, 81315 (1981)

[23] Saddoughi S. G., \& Veeravalli S. V., Local isotropy in turbulent boundary layers at high Reynolds number, J. Fluid Mech., 268, 333-372 (1994)

[24] Sengupta T. K., High Accuracy Computing Methods: Fluid Flows and Wave Phenomena, Cambridge Univ. Press, USA (2013)

[25] Sengupta T. K., A critical assessment of simulations for transitional and turbulent flows, in IUTAM Symp. Proc. Advances in Computation, Modeling and Control of Transitional and Turbulent Flows, 491-532 (2016)

[26] Sengupta T. K., \& Bhaumik S., Onset of turbulence from the receptivity stage of fluid flows, Phys. Rev. Lett., 107(15), 154501 (2011)

[27] Sengupta T. K., Bhaumik S., \& Bhumkar Y. G., Direct numerical simulation of twodimensional wall-bounded turbulent flows from receptivity stage, Phys. Rev. E, 85(2), 026308 (2012)

[28] Sengupta T. K., \& Bhole A., Error dynamics of diffusion equation: Effects of numerical diffusion and dispersive diffusion, J. Comp. Phys., 266, 240-251 (2014)

[29] Sengupta T. K., Dipankar A., \& Sagaut P., Error dynamics: Beyond von Neumann analysis, J. Comp. Phys., 226(2), 1211-1218 (2007)
[30] Sengupta T. K., Singh H., Bhaumik S., \& Chowdhury R. R., Diffusion in inhomogeneous flows: Unique equilibrium state in an internal flow, Computers \& Fluids, 88, 440-451 (2013)

[31] Skote M., \& Henningson D. S., Direct numerical simulation of a separated turbulent boundary layer, J. Fluid Mech., 471, 107-136 (2002)

[32] Smith L. M., \& Yakhot V., Finite-size effects in forced two-dimensional turbulence, J. Fluid Mech., 274, 115-138 (1994)

[33] Suman V. K., Sengupta T. K., Jyothi Durga Prasad C., Surya Mohan K., \& Sanwalia D., Spectral analysis of finite difference schemes for convection diffusion equation, Computers \& Fluids, 150, 95-114 (2017)

[34] Suman V. K., Sengupta T. K., \& Mathur J. S., Effects of numerical anti-diffusion in closed unsteady flows governed by two-dimensional Navier-Stokes equation, Computers \& Fluids, 201, 104479 (2020)

[35] Tan R., Ooi A., \& Sandberg R. D., Twodimensional analysis of hybrid spectral/ finite difference schemes for linearized compressible Navier-Stokes equations, J. Sci. Comp., 87(2), 42 (2021)

[36] von Neumann J., \& Richtmyer R. D., On the numerical solution of partial differential equations of parabolic type, Los Alamos Rept., Series A, LA-657, 1-17 (1947)

[37] Yeung P. K., Donzis D. A., \& Sreenivasan K. R., Dissipation, enstrophy and pressure statistics in turbulence simulations at high Reynolds numbers, J. Fluid Mech., 700, 5-15 (2012) 


\section{A Appendix}

The first step in performing global spectral analysis for a numerical scheme is to obtain its complex numerical amplification factor $G_{N u m}$. Here, we provide $G_{N u m}$ for RK2-, RK3- and RK4Fourier spectral methods for the linear convectiondiffusion equation. The derivation is based on the global spectral analysis for general finite difference schemes for the model linear convection and convection-diffusion equations provided in [24, 33]. The interested reader should consult these references for complete details.

Following the approach in [33], the complex numerical amplification factor for RK2-, RK3- and RK4-Fourier spectral methods is given as

$$
\begin{aligned}
& G_{\text {Num }, R K 2}=1-B+\frac{B^{2}}{2} \\
& G_{\text {Num }, \text { RK } 3}=1-B+\frac{B^{2}}{2}-\frac{B^{3}}{6} \\
& G_{\text {Num }, R K 4}=1-B+\frac{B^{2}}{2}-\frac{B^{3}}{6}+\frac{B^{4}}{24}
\end{aligned}
$$

where $B=P e(k \Delta x)^{2}+\mathrm{i} N_{c}(k \Delta x)$, with $N_{c}$ and $P e$ denoting the CFL and Peclet numbers, respectively.

From the complex amplification factors given above for the RK2-, RK3- and RK4-Fourier spectral methods, the numerical diffusion coefficient $\left(v_{N u m}\right)$, phase speed $\left(c_{N u m}\right)$ and group velocity $\left(V_{g, N u m}\right)$ are obtained as

$$
\begin{aligned}
\frac{v_{\text {Num }}}{v} & =-\frac{\ln (|G|)}{(k \Delta x)^{2} P e} \\
\frac{c_{\text {Num }}}{c} & =-\frac{1}{(k \Delta x) N_{c}} \tan ^{-1}\left[\frac{\mathfrak{I}(G)}{\mathfrak{R}(G)}\right] \\
\frac{V_{g, \text { Num }}}{V_{g, \text { Phys }}} & =-\frac{1}{N_{c}} \frac{d}{d(k \Delta x)}\left(\tan ^{-1}\left[\frac{\mathfrak{I}(G)}{\mathfrak{R}(G)}\right]\right)
\end{aligned}
$$

where $G$ takes $G_{N u m, R K 2}$ or $G_{N u m, R K 3}$ or $G_{N u m, R K 4}$ depending on the scheme chosen for analysis.

Contribution of individual authors to the creation of a scientific article (ghostwriting policy)

Conceptualization and supervision: Tapan K Sengupta

Analysis, investigation and visualization: Vajjala K Suman and Prasannabalaji Sundaram.

Writing, reviewing and editing: Tapan K Sengupta, Aditi Sengupta, Vajjala K Suman, Prasannabalaji Sundaram

Sources of funding for research presented in a scientific article or scientific article itself

This study did not receive funding of any form.

\section{Creative Commons Attribution License 4.0 (Attribution 4.0 International, CC BY 4.0)}

This article is published under the terms of the Creative Commons Attribution License 4.0

https://creativecommons.org/licenses/by/4.0/deed.en_US 